\title{
MODELO PROSPECTIVO DEL SERVICIO DE TRANSPORTE AÉREO VERTICAL URBANO EN LA CIUDAD DE LIMA PARA EL DESARROLLO ESTRATÉGICO NACIONAL
}

\author{
José Ricardo Gutiérrez Rivas ${ }^{1}$
}

\section{Resumen}

Como resultado de la Fase Final de Maestría de Prospectiva Estratégica para el Desarrollo Nacional de la Universidad Mayor de San Marcos, se presentó la Tesis del Modelo Prospectivo del Servicio de Transporte Aéreo Vertical Urbano en la ciudad de Lima para el desarrollo estratégico nacional del Perú, con el fin de contribuir con el Estado peruano a fin de que en un futuro no muy lejano la ciudadanía pueda disponer de una flota de aeronaves de despegue vertical en cantidad y capacidad suficiente para afrontar la demanda en tiempo de paz y de emergencias en caso que éstas se presenten evitando el caos por desastres naturales o producidos por el hombre.

El objetivo del presente trabajo, es forjar un precedente que facilite la aplicación de la prospectiva estratégica para el desarrollo nacional en el sector aeronáutico en el Perú.

El problema: ¿Cuán importante es el escenario futurible relacionado con la capacidad de respuesta del estado para el transporte aéreo vertical urbano tanto en tiempo de paz como en las emergencias en la Ciudad de Lima Metropolitana?

El presente estudio se basó en el empleo de la técnica de recolección de datos del tipo de entrevistas abiertas y cerradas a diferentes grupos de expertos (autoridades de aeronáutica, instituciones civiles relacionadas: OSINERGMIN, INDECI, Municipalidad de Lima y expertos pilotos de helicópteros.

Se realizó el análisis posterior mediante el empleo de diferentes herramientas técnicas de prospectivas tales como la de Peter Schwartz, Foresight y del método de Godet, que permitieron el planteamiento de los escenarios futuribles y las estrategias correspondientes para su consecución.

Se evaluaron las respuestas y se confeccionó varios matrices relacionadas a las diferentes herramientas de prospectiva, para identificar las variables clave. 
Las variables clave se relacionan de acuerdo a su importancia y conflictividad, se identificaron las positivas más relevantes y las negativas más obsoletas, que fundamentan la confección de escenarios.

Se obtuvieron cómo resultado cuatro escenarios:

1. «Two Thumbs Up»: Con la más óptima Tecnología y sostenibilidad promovido por el estado y apoyado por el mercado.

2. «Buscando Estoy»: El Estado da las condiciones parciales de facilitación para la operación de transporte aéreo vertical.

3. «Helicópteros Raca»: El Estado pone muchas barreras, pero promueve el turismo.

4. «Me quedo en Tierra»: El Estado pone muchas barreras al desarrollo del Transporte Aéreo Vertical Urbano.

Palabras claves: Prospectiva Estratégica, vuelo vertical urbano, helicópteros, desarrollo nacional, Lima.

\section{PROSPECTIVE MODEL OF VERTICAL URBAN AIR TRANSPORT SERVICE IN THE CITY LIMA FOR NATIONAL STRATEGIC DEVELOPMENT}

\section{Abstract}

As a result of the Final phase of mastery of strategic foresight for the national development taken in the de San Marcos Mayor University, I presented the thesis: Prospective Model of Vertical Air Transport Service in the City of Lima for the Peru National Strategic Development. Hoping to contribute to the Peruvian Government so that in the not-too-distant future the citizens can have a vertical take-off aircraft fleet with quantity and sufficient capacity to deal with demand in peace and emergencies time in case that these occur preventing chaos by natural disasters or human.

The objective of this work is to forge a precedent for the help to apply the strategic foresight for national development in the aeronautical sector in the Peru.

The problem: How important is the future scenario related to the ability of the State's response to urban vertical air transport both in peacetime and in emergencies in the Metropolitan City of Lima?

The present study was based on the use of the technique of data collection of the type of interviews open and closed to different groups of experts (aeronautical authorities, civil institutions: OSINERGMIN, INDECI, Lima Municipality and expert pilots of helicopters.)

The subsequent analysis using different prospective tools techniques such as Peter Schwartz, Foresight and Godet methods, which allowed the approach of potential scenarios and corresponding strategies for their achievement. 
The responses were evaluated and several matrixes related to different foresight tools, to identify the key variables.

The key variables are related according to their importance and conflicts, the most relevant positive and negative most obsolete, that underlie the preparation of scenarios were identified.

As a result were obtained four scenarios:

1. Two Thumbs Up": with the most optimal technology and sustainability promoted by the State and supported through the market.

2. "Looking For": the State gives the partial terms of facilitation for vertical air transport operation.

3. "Helicopters Raca": the State puts many barriers, but promotes tourism.

4. "I Stay on Ground": the State puts many barriers to the development of Urban Vertical Air Transport"

Keywords: Strategic Foresight, urban vertical flight, Helicopters, national development, Lima.

\section{Situación general}

\section{Antecedentes}

En el Perú por diversos motivos existe un limitado número de pilotos de helicópteros. No hay escuela de pilotaje y los pocos helicópteros de las FFAA y FFPP que existen, se encuentran apoyando a las operaciones en el VRAEM, mientras que los helicópteros civiles de aproximadamente 14 compañías operadoras apoyan las operaciones petroleras. Asimismo, es notorio que en Lima a pesar de la existencia de la Reglamentación de la Ley de Aeronáutica (Congreso de la República del Perú, 2000; Ministerio de Transportes y Comunicaciones, 2010; Ministerio de Transporte y Comunicaciones, 2001; Presidente de la República, 2006) no se realiza el servicio de vuelo vertical urbano, desperdiciándose la oportunidad de empleo del espacio aéreo disponible, a pesar de que se tiene una tendencia creciente de la población y de la congestión vehicular que ocasiona gran pérdida de tiempo, combustible y genera polución.
No se conocen trabajos de investigación prospectiva precedentes a éste tema a nivel internacional y nacional, pero en algunas ciudades del mundo moderno, (Ej. Nueva York, Sao Paulo, Argentina y Paraguay) ya se emplean los servicios de transporte aéreo vertical sobre las ciudades, permitiendo la generación de empleo y el desarrollo de la industria y cultura aeronáutica. En el caso de la ciudad de Sao Paulo con una población de aproximadamente, 20 Millones de personas en la actualidad cuentan de una manera muy organizada el servicio de transporte aéreo vertical implementado según comentó el Presidente de la Asociación de Pilotos de Helicópteros del Brasil, con una flota de 600 helicópteros y realizando aproximadamente 550 operaciones diarias (Rodrigo Bravo, 2012). 


\section{Planteamiento del problema}

Planteamiento del Problema: ¿Cuán Importante es el escenario futurible de la capacidad de respuesta del Estado en un desastre nacional con respecto al empleo del servicio de transporte de aéreo vertical urbano en Lima Metropolitana?

Problemas Secundarios:

- ¿Cómo determinar quiénes son los actores en el escenario futurible a proponer?

- ¿Cuáles son las principales variables o drivers claves para el escenario futurible?

- ¿Cuáles son los lineamientos a seguir para lograr el escenario futurible?

\section{Justificación teórica de la investigación}

La presente investigación dará como resultado escoger los mejores cursos de acción que se puedan tomar a fin de poder aprovechar el empleo del espacio aéreo tal como se hace en las ciudades más modernas a nivel mundial, a fin de que sea aprovechado a favor de los diferentes sectores creando una sinergia de beneficios para los diferentes actores, uno de ellos es la oferta oportuna de transporte de personas y carga, así como la creación de una constante fuente de generación de trabajo y cómo consecuencia, lograr disponer de una flota importante que permita disponer de la capacidad de respuesta requerida para afrontar las situaciones de emergencia.

\section{Amenaza de catástrofes en perú}

El Perú tiene una gran vulnerabilidad (WilchesChaux, 2001), está amenazado constantemente por los efectos de los fenómenos naturales tales como sismos, fenómenos meteorológicos, catástrofes ecológicas, epidemias, etc. o también por posibles desastres ocasionados por el hombre (incendios, explosiones, contaminación, etc.).

El Estado peruano, al no contar con los medios de transporte aéreo vertical en la ciudad de Lima, no dispone de la capacidad de proporcionar una respuesta inmediata de apoyo a la población en caso ocurran desastres ((Wilches-Chaux, 2001) de grandes proporciones, lo cual puede ocasionar en la población afectada una crisis que si no es tratada adecuadamente se puede convertir en un caos incontrolable pudiendo resultar en más víctimas por la falta de previsión.

\section{Objetivos principales de la investigación}

Determinar la importancia de la formulación de un escenario futurible en la capacidad de respuesta del Estado en época de paz para la promoción de la conectividad y riqueza, así como en época de emergencia ante un desastre nacional en relación al empleo del servicio de transporte vertical urbano en Lima Metropolitana.

Nota: Se plantea el requerimiento del objetivo principal que traerá como consecuencia los objetivos específicos.

\section{Objetivos específicos}

1. Establecer las variables o drivers para el escenario futurible a ser propuesto.

2. Priorizar las variables.

3. Identificar los Escenarios futuribles.

4. Determinar los actores de escenario futurible propuesto.

5. Establecer las variables o drivers para el escenario futurible a ser propuesto.

6. Determinar las maneras de orientar el flujo de los esfuerzos de los actores que permitan el desarrollo auto sostenido de las operaciones de transporte aéreo vertical urbanos

\section{Hipótesis}

\section{Hipótesis general}

El diseño de un escenario posible (futurible) tiene una importancia gravitante en la capacidad de respuesta del Estado en un desastre nacional con respecto al empleo del Servicio de Transporte Aéreo Vertical Urbano. 


\section{Hipótesis específicas \\ HS1}

Mediante un método basado en la opinión de expertos se determinan los principales actores en los escenarios futuribles a proponer.

\section{HS2}

Las variables relacionadas al ámbito político, económico, social y tecnológico son claves para los escenarios futuribles a proponer.

\section{HS3}

Los lineamientos orientados en el ámbito normativo, de infraestructura, educativo y económicos son requeridos para lograr los escenarios futuribles.

\section{Identificación de Variables de Dependencia VD1:}

Nivel de importancia en la capacidad de respuesta del Estado en un desastre en Lima Metropolitana con respecto al empleo.

VD2:

Resultados del método basado en expertos.

VD3:

Identificación de variables claves.

VD4:

Identificación de los lineamientos a seguir.

\section{Matriz de Consistencia [7]}

Permite a la presente investigación diseñar un camino adecuado y pragmático para solucionar los diferentes factores críticos de éxito y permite llegar a construir los escenarios futuribles que se acerquen a lo más deseable. Se establece la pregunta problemática general: ¿Cuán importante es el escenario futurible de la capacidad de respuesta del Estado Peruano sobre su capacidad de respuesta ante un desastre nacional con respecto al empleo del servicio de helicópteros urbanos en Lima Metropolitano?

En el siguiente cuadro 01, se puede apreciar la Matriz de Consistencia, la cual especifica los problemas encontrados con su justificación, objetivos con hipótesis por cara problema, variables e indicadores por cada hipótesis.

\section{Cuadro 01 Matriz de Consistencia}

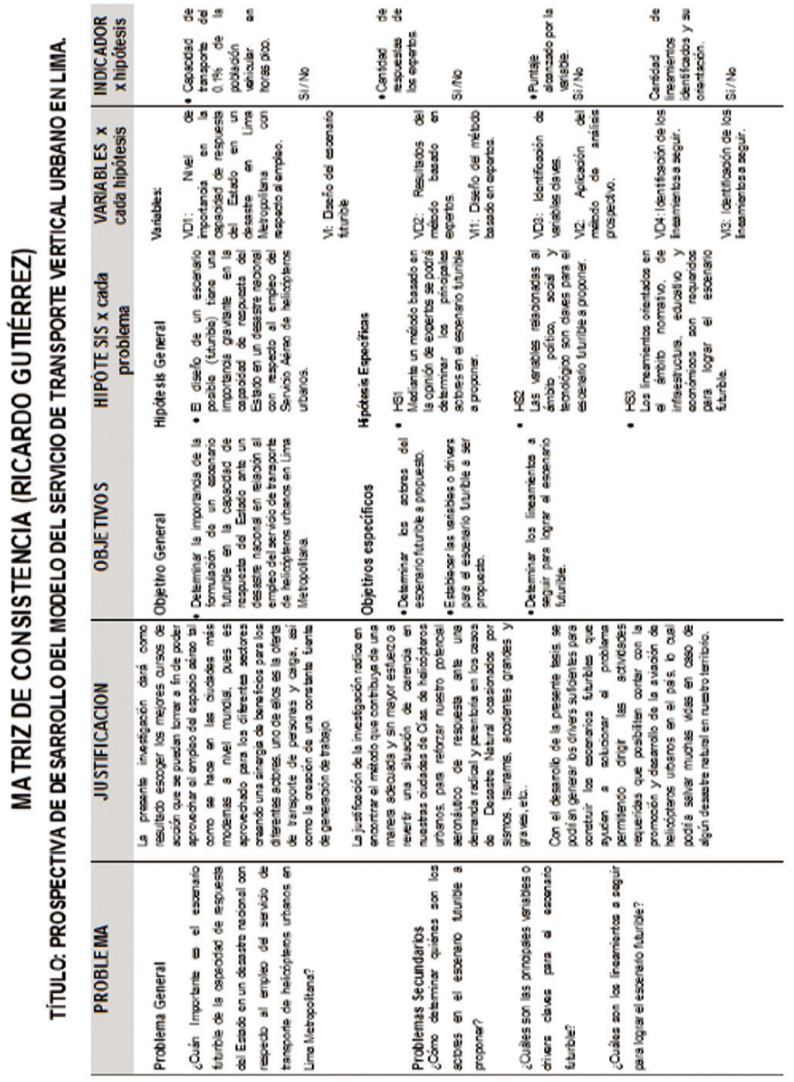

\section{Marco teórico}

\section{Antecedentes de la investigación}

Se hizo un listado de los antecedentes de la investigación, en el que se tomaron en cuenta las diferentes obras referentes que fortalecen y dan sustento a las bases del presente trabajo de investigación, tales como:

1. Manual del Helicóptero de Combate de la FAP (Gutiérrez, 1982).

2. InkaKopter, Transporte Aéreo de Turismo VIP (Gutiérrez, y Carbonel, 2011).

3. Creación de la Escuela de Helicópteros Helischool (Gutiérrez, 2010)

4. Los ricos de Sao Paulo esquivan el tráfico con Helicópteros (CNN, 2010)

5. América Latina un Mercado en Ascenso para los helicópteros (Harrison, 2013) 


\section{Marco legal}

Se listaron las diferentes Leyes y Reglamentos relativos al tema de investigación, notándose que existe un gran vacío legal en lo relacionado a las operaciones de vuelo vertical urbano por parte del Ministerio de Transportes y Comunicaciones (MTC), Instituto de Defensa Civil (INDECI), Congreso de la República, Regiones y Municipios.

\section{Metodología empleada para el análisis de las Variables:}

Se inició el trabajo de campo, con citas a varios grupos de expertos ligados a la aeronáutica y a los diferentes escenarios relacionados a dicho sector.

\section{Entrevistas y Encuestas Delphi [13]}

Tiene como objetivo, partiendo de la consulta a expertos y gracias a cuestionarios sucesivos, poner de relieve convergencias de opinión y despejar ciertos consensos sobre temas precisos. El objetivo más frecuente de la encuesta Delphi es hacer llegar la luz que aportan determinados especialistas sobre las zonas de incertidumbre de determinado tema para tomar así mejores decisiones.

Para tal fin se utilizaron diferentes ocasiones de trabajo, tales como seminarios, entrevistas y encuestas privadas con la finalidad de poder identificar las diferentes variables, cuyas sumatorias darían resultados que ayudarían a seleccionar posteriormente con el empleo de diferentes técnicas las más importantes y las más gobernables, los impactos entre ellas y su motricidad.
Se efectuaron las visitas de campo para realizar encuestas con los diferentes grupos de expertos, lográndose confeccionar diversas matrices comparativas y estadísticas que resultaron luego de un análisis estructural de las variables, en la identificación de las variables de más importancia (las más votadas) requeridas para realizar la Matriz de Motricidad para identificar su dependencia, estableciéndose los pesos de influencia entre las variables.

\section{Método STEEP-VOC [14]}

Para la selección y validación de los factores de cambio (variables) se emplea la metodología de encuestas Delphi a los expertos, el método STEEP-VOC que relacionan las variables con el tema social, tecnológico económico, medio ambiental, actitudes y valores políticos.

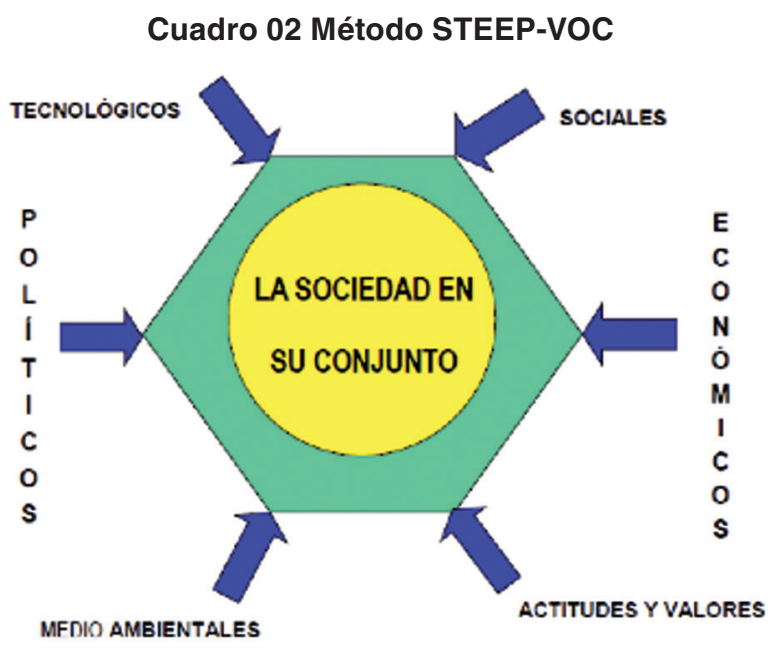

\section{Matriz Porcentual de Variables [15]}

Una vez obtenidas la identificación de variables, se utilizó la Matriz Porcentual de Variables con la cual se logró priorizar las variables claves del estudio. 


\section{CUADRO 03. Matriz Porcentual de las Variables}

\begin{tabular}{|c|c|c|c|c|}
\hline Orden & Variable & Total & Porcentaje & Acumulado \\
\hline 6 & $\begin{array}{l}\text { 1) Demográico: Explosión esporada en el alcance dol } \\
\text { estuda }\end{array}$ & 102 & $9.3 \%$ & $9.3 \%$ \\
\hline 11 & $\begin{array}{l}\text { e) Turismo: Afuencia de person as de otros paises y a nivel } \\
\text { del pais }\end{array}$ & 102 & $9.3 \%$ & $18.6 \%$ \\
\hline 12 & if) Tratados de Libre Comercio. Condiciones y tormas & 102 & $9.3 \%$ & $27.8 \%$ \\
\hline 18 & 1) Eficiencla del Aparato Estatal: Permisos y ucencias & 102 & $9.3 \%$ & $37.1 \%$ \\
\hline 19 & $\begin{array}{l}\text { a) Nueva Tecndoga de Helićópteros Major eficiencia y } \\
\text { rentabilidad debido a la tecnologia }\end{array}$ & 102 & $9.3 \%$ & $46.4 \%$ \\
\hline 23 & $\begin{array}{l}\text { e) Infacstructura para aterrizaj: Contar con pads, } \\
\text { nelipuertos irfraestructura para el servicio }\end{array}$ & 102 & $9.3 \%$ & $55.7 \%$ \\
\hline 24 & $\begin{array}{l}\text { A) Abastecimicrto do hsumos para la } \\
\text { industria Combustible, partes y piezas }\end{array}$ & 102 & $9.3 \%$ & $65.0 \%$ \\
\hline 3 & $\begin{array}{l}\text { c) Formalicad en el servicio de transporte: Nuevas reglas } \\
\text { gue se exigen en a los transportistas adecuarse }\end{array}$ & 36 & $33 \%$ & $68.2 \%$ \\
\hline 15 & $\begin{array}{l}\text { c) Nivel de Trarsparencia: Conocimiento de los } \\
\text { presuouestos y ejecución }\end{array}$ & 35 & $3.2 \%$ & $71.4 \%$ \\
\hline 2 & $\begin{array}{l}\text { b) Saluración del transporte terrestre (Congestionamiento): } \\
\text { Un aumento del parque automotor debido a las facilidades }\end{array}$ & 30 & $27 \%$ & $74.2 \%$ \\
\hline 4 & d) Contamineciớn Nueles de Polución y Ruico & 29 & $26 \%$ & $76.8 \%$ \\
\hline 10 & $\begin{array}{l}\text { d) Consumo der cápita: Consumo en Bs y Ss de la } \\
\text { población }\end{array}$ & 28 & $25 \%$ & $79.3 \%$ \\
\hline 17 & e) Manejo Tributaio. Principales escudos fiscales & 28 & $25 \%$ & $81.9 \%$ \\
\hline 1 & a) Estilo de Vida: Debido a que el nuevo servicio cambiaria & 27 & $25 \%$ & $84.3 \%$ \\
\hline 13 & $\begin{array}{l}\text { a) Inclus ión Social: Canticad de personas que accesar al } \\
\text { servicio }\end{array}$ & 26 & $24 \%$ & $96.7 \%$ \\
\hline 20 & $\begin{array}{l}\text { D) Competencia de M ano de Obra: Contar con Técnicas y } \\
\text { Pilotos calicados }\end{array}$ & 26 & $24 \%$ & $89.1 \%$ \\
\hline 7 & $\begin{array}{l}\text { a) Ingresos per cápta Nivel de sueldos y salarios de la } \\
\text { población }\end{array}$ & 23 & $21 \%$ & $91.2 \%$ \\
\hline 16 & d) Regulación Gubernamental: Tratamierto de las leyes & 23 & $21 \%$ & $93.3 \%$ \\
\hline 21 & $\begin{array}{l}\text { c) Acceso a la tecnol ogia de intormación. Benchmarking } \\
\text { de los modelos de transporte }\end{array}$ & 19 & $1.7 \%$ & $95.0 \%$ \\
\hline 5 & $\begin{array}{l}\text { e) Migración a la Urie: Desplaz amiento de las personas } \\
\text { de provincias a la capital }\end{array}$ & 18 & $1.6 \%$ & $96.6 \%$ \\
\hline 9 & $\begin{array}{l}\text { c) Disponiblildad de Crédilos: Lineas de créditos otorgadas } \\
\text { por el sistema inanciero }\end{array}$ & 18 & $1.6 \%$ & $96.3 \%$ \\
\hline 8 & b) Comercio exterior: Nveles de Ex portación e Importación & 7 & $0.6 \%$ & $98.9 \%$ \\
\hline 14 & b) Democracia participativa: Patticipación en las leyes & 7 & $0.6 \%$ & $99.5 \%$ \\
\hline 22 & $\begin{array}{l}\text { d) Investigación y Dosarallo del modelo de } \\
\text { negocios. Aplicación de mejores prácticas en el negocio }\end{array}$ & 5 & $0.5 \%$ & $100.0 \%$ \\
\hline & TOTALES & 1099 & $100.0 \%$ & \\
\hline
\end{tabular}

\section{Identificación de actores Método MACTOR [16]}

Usando el método MACTOR sin el empleo de software, permitió identificar a los actores más importantes incluyendo el grado de influencias entre ellos respecto a su impacto en el sistema.
1. MTC-DGAC

2. Compañías de Helicópteros

3. OSINERGMIN

4. Población

5. DINANDRO

6. Competencia del Transporte Terrestre.

\section{Cuadro 04: Identificación de Actores}

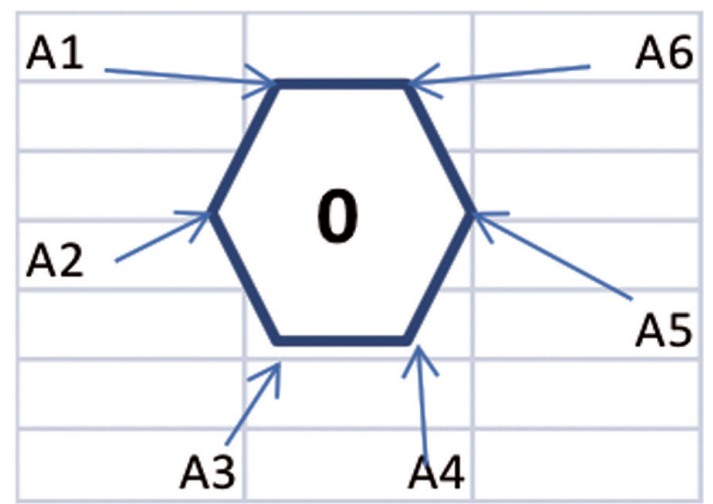

\section{Matriz de Posiciones de Actores por Objetivos (IMAO)}

En el Cuadro 05 siguiente se puede visualizar la Matriz de las Posiciones de Actores por Objetivos (IMAO), en el cual se interrelacionan a los diferentes actores con los objetivos, a fin de poder establecer las dependencias unos de otros y de ésta forma poder establecer su correspondencia posteriormente para la elaboración de los escenarios futuribles.

CUADRO 05. Matriz de las Posiciones de Actores por Objetivos (IMAO)

\begin{tabular}{|c|c|c|c|c|c|c|c|c|c|}
\hline & \multicolumn{8}{|c|}{ IIAAO: Matriz de las Posiciones de actores x Objetivos } & \\
\hline & & & \multirow{4}{*}{ 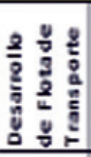 } & \multirow{4}{*}{ 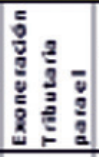 } & \multirow{4}{*}{ 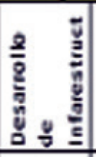 } & \multirow{4}{*}{ 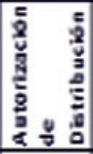 } & \multirow{4}{*}{ 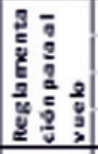 } & & \\
\hline & & & & & & & & & \\
\hline & & & & & & & & & \\
\hline & & & & & & & & & \\
\hline & & & 01 & 02 & 03 & 04 & 06 & S+ & S. \\
\hline & MTC (DGAC) (Reglamentación) & A1 & 1 & 1 & 1 & 0 & 1 & 4 & 0 \\
\hline & Comparíss de Helcóoteros & $\mathrm{A2}$ & 1 & 0 & 1 & 1 & 1 & 4 & 0 \\
\hline 3 & OSINERGMIN(Fuel) & $\mathrm{A3}$ & 1 & 1 & 0 & 1 & 1 & 4 & 0 \\
\hline 4 & Pobleción & A4 & 0 & -1 & -1 & 0 & 0 & 0 & -2 \\
\hline 5 & DINANDRO & $\mathbf{A 5}$ & -1 & 0 & 0 & -1 & 0 & 0 & -2 \\
\hline & Competencia (Transporte Terrestre) & $A B$ & -1 & -1 & -1 & 0 & 0 & 0 & .3 \\
\hline & & S+ & 3 & 2 & 2 & 2 & 3 & & \\
\hline & & s. & .2 & -2 & -2 & -1 & 0 & & \\
\hline
\end{tabular}


Con la Matriz de Posiciones de Actores por Objetivos (IMAO), se establece una evaluación de dependencia entre los actores con las variables requeridas para la construcción de escenarios. Observándose que la posición de mayor importancia la tienen los actores con respecto a los objetivos, del MTC-DGAC, de las compañías de helicópteros y la de OSINERGMIN.

\section{Relación de Objetivos y Matriz de Actor, Actor (MAA)}

En el cuadro 06 siguiente, se muestra los siguientes objetivos que ayudarán a identificar la motricidad de los actores la Matriz de Actor, Actor (MAA).

\section{Cuadro 06. Objetivos}

\begin{tabular}{|l|l|}
\hline \multicolumn{3}{|c|}{ Objetivos } \\
\hline 01 & Desarrollo de Flota de Transporte Aćreo Vertical para la paz y emergencias \\
\hline $\mathbf{O 2}$ & Exone ración Tributaria para el Tran sporte Aéro Vertical Urbano en Lima \\
\hline $\mathbf{O 3}$ & Desarrollo de Infarestructura (Constriucción de Helipads \\
\hline $\mathbf{0 4}$ & Autorizadión de Distribución de Combustible de aviación (Turbo A-1) \\
\hline $\mathbf{O 5}$ & Reglamentación para al vuelo urbano. \\
\hline
\end{tabular}

El Cuadro 07 siguiente, se tiene la Matriz que relaciona los actores con los diferentes actores (MAA).

CUADRO 07. Relación de Objetivos y la Matriz de Actor, Actor (MAA)

\begin{tabular}{|r|l|c|c|c|c|c|c|c|}
\hline & Matriz Actor, Actor (MAA) & & A1 & A2 & A3 & A4 & A5 & A6 \\
\hline 1 & MTC (DGAC) (Reglamentación) & A1 & & $+3,0$ & $+3,0$ & $0,-2$ & $0,-2$ & $0,-3$ \\
\hline 2 & Compañilias de Helicópteros & $\mathrm{A} 2$ & $+3,0$ & & $+3,0$ & $0,-1$ & $0,-1$ & $0,-2$ \\
\hline 3 & OSINERGMIN (Fuel) & $\mathrm{A} 3$ & $+3,0$ & $+3,0$ & & $0,-1$ & $0,-2$ & $0,-2$ \\
\hline 4 & Población & $\mathrm{A} 4$ & $0,-2$ & $0,-1$ & $0,-1$ & & 0,0 & 0,0 \\
\hline 5 & DINANDRO & $\mathrm{A} 5$ & $0,-2$ & $0,-1$ & $0,-2$ & 0,0 & & 0,0 \\
\hline 6 & Competencia (Transporte Terrestre) & $\mathrm{A} 6$ & $0,-3$ & $0,-2$ & $0,-2$ & 0,0 & 0,0 & \\
\hline
\end{tabular}

En ésta matriz se comparan los valores de dependencias de los diferentes actores identificados, lográndose darles valores de motricidad (Mi) y Dependencia (Di). Pudiéndose observar los diferentes valores de relación entre los mismos.

\section{Matriz de Niveles de Influencias}

A continuación en el cuadro 08 siguiente, se relacionan los diferentes actores unos a otros y se obtienen unos valores de las influencias entre ellos, para lo cual se listó una gama de valores de menor a mayor del 0 al 3 de acuerdo a lo siguiente:

Tablero de actores $\mathrm{x}$ actores

Escala de Influencia Potencial:

0 Nula

1 Débil

2 Media

3 Fuerte 
CUADRO 08. Matriz de Niveles de Influencias

\begin{tabular}{|c|c|c|c|c|c|c|c|c|c|c|}
\hline \multicolumn{4}{|c|}{ Cuales el nivelde influencias entre el $A 1$ y el $A 2$} & & & & & & \multirow[b]{2}{*}{ MOTRIC } & \multirow[b]{2}{*}{ IDAD (Mi) } \\
\hline & Matriz de Acciones Directas & & A! & $A 2$ & A3 & A4 & A5 & $A B$ & & \\
\hline 1 & MTC (DGAC) (Reglamentación) & $\mathrm{A} 1$ & & 3 & 1 & 2 & 1 & 3 & 10 & \\
\hline 2 & Compañís de Helicópteros & $\mathrm{A} 2$ & 2 & & 2 & 1 & 0 & 0 & 5 & \\
\hline 3 & OSINERGMIN (Fuel) & $A 3$ & 1 & 3 & & 3 & 1 & 2 & 10 & \\
\hline 4 & Población & A4 & 3 & 1 & 2 & & 1 & 3 & 10 & \\
\hline 5 & DINANDRO & A5 & 1 & 3 & 3 & 2 & & 2 & 11 & \\
\hline 6 & Competencia (Trans porte Terrestre) & $\mathrm{A} \theta$ & 3 & 1 & 1 & 2 & 1 & & 8 & \\
\hline & DEPENDENCIA (Di) & & & & & 1 & 4 & 10 & & 54 \\
\hline
\end{tabular}

En éste cuadro de identificación de Motricidad, se puede evaluar el nivel de influencias y la motricidad entre los diferentes actores.

En el Cuadro 09 siguiente, se identifican con valores la influencia de los diferentes actores.

CUADRO 09. De Identificación de Influencia de los Actores

\begin{tabular}{|c|c|c|}
\hline & ri & $\mathrm{r}^{*}$ (normalizado) \\
\hline MTC (DGAC) (Reglamentación) & 0.09 & 1.05 \\
\hline Compañias de Helicópteros & 0.03 & 0.33 \\
\hline OSINERGMIN (Fuel) & 0.10 & 1.11 \\
\hline Población & 0.09 & 1.05 \\
\hline DINANDRO & 0.15 & 1.70 \\
\hline Competencia (Transporte Terrestre) & 0.07 & 0.75 \\
\hline Promedio & 0.09 & 6.00 \\
\hline
\end{tabular}

Se puede deducir que la mayor influencia de los actores está en la DINANDRO, y le sigue la OSINERGMIN.

Cabe resaltar que estos dos actores tienen que ver con el sistema de abastecimiento de combustible de aviación, que actualmente es considerado como insumo químico precursor para la elaboración de la pasta básica de cocaína, pero es básico que sin combustible no se puede operar en la aeronáutica.

En el Cuadro 10 siguiente, se observa gráficamente los valores de influencia de cada variable.

\section{CUADRO 10. Influencia de Actores}

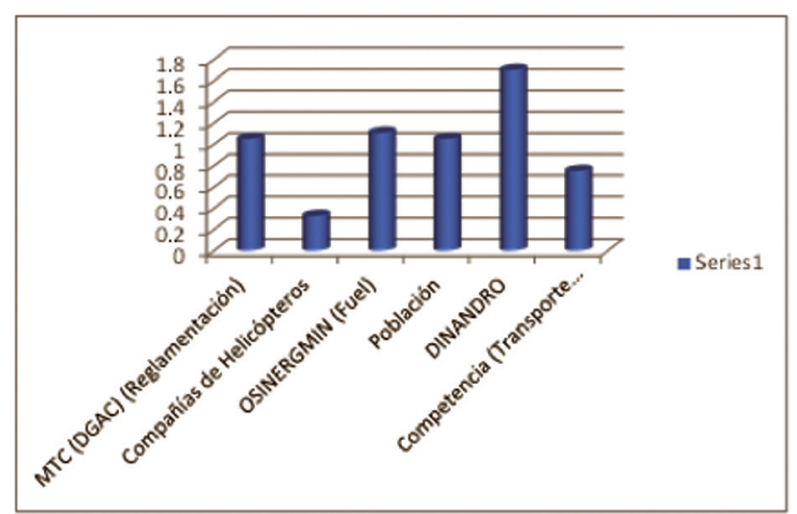

Con ésta matriz se puede identificar gráficamente, los actores que tienen una mayor influencia en las dependencias de las variables. Pudiéndose observar que la DINANDRO cuenta con el mayor peso comparado con el de los demás actores.

\section{Identificación de Alianzas y Conflictos}

Entre los diferentes actores, se pueden producir relaciones que favorecen y pueden contribuir a formar alianzas, así como también pueden producir incompatibilidades y generar conflictos entre sí.

En el cuadro siguiente, se puede observar de manera gráfica la relación entre de alianzas y/o conflictos entre los diferentes actores que están interconectados en los escenarios 
CUADRO 11. De Identificación de Alianzas y Conflictos

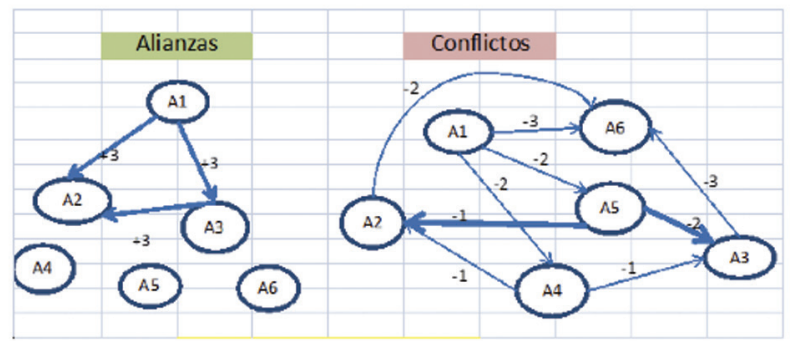

Con el cuadro anterior se puede visualizar gráficamente las alianzas y los conflictos generados entre los diferentes actores con las dependencias de las variables.

\section{Creación de escenarios [17]}

Una vez que se efectuaron las visitas de campo para realizar encuestas con los diferentes grupos de expertos, lográndose confeccionar diversas matrices comparativas y estadísticas que resultaron luego de un análisis estructural de las variables, en la identificación de las variables de mayor importancia (las más votadas) requeridas para realizar la Matriz de Motricidad para identificar su dependencia, estableciéndose los pesos de influencia entre las variables.

Se estableció la identificación de actores y se compararon en la matriz IGO [18] con las variables a fin de establecer qué actores tenían más importancia y gobernabilidad sobre las variables, lo cual favorecería posteriormente a la construcción de los escenarios.

Luego se determinó la conflictividad de las variables, las cuales pueden ser con impactos directos o indirectos, permitiendo luego establecer las acciones a fin de poder contrarrestar o minimizar dichos impactos.

Una vez identificadas las dependencias, y el nivel de conflictividad entre las variables, se procedió a realizar la construcción de cuatro escenarios, que se evaluaron usando el método de las «Cáscaras de Plátano». Dicho método se caracteriza en colocar los escenarios en un gráfico de coordenadas cartesianas a fin de ubicar los puntos de conflicto a fin de poder evaluar su posibilidad de realización.

Figura 12. Método «Cáscaras de Plátano»

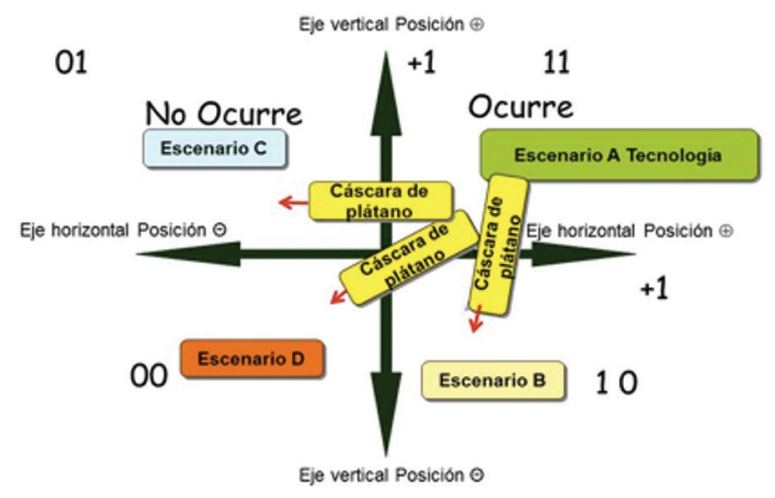

\section{Escenarios elegidos}

Se eligieron cuatro escenarios de acuerdo a lo siguiente:

\section{«Two Thumps Up»}

En la que se consiguen completar los factores facilitadores a fin de que las aeronaves de transporte aéreo vertical urbano, puedan operar en forma organizada y segura, ofreciendo un servicio al mercado en un ambiente considerado de «Océano Azul».

Se promueve de manera multisectorial la Cultura Aeronáutica en la población; facilitando la organización del sistema con la creación del órgano de control, de las empresas aéreas de vuelo urbano con aeronaves modernas y seguras, helipuertos, desarrollo de clústeres aéreos (Escuelas, Servicios de Operaciones y de Mantenimiento), se establecen rutas o carreteras aéreas con helipuertos alternos en parques, canchas deportivas, centros comerciales, hospitales, carreteras principales, edificios, etc.

Con beneficios tributarios se promueve inversión de las empresas de transporte vertical y 
por ende la generación de empleo en el sector de aeronáutica llegando a conseguir un ritmo de crecimiento sostenido en el mercado. Las empresas de helicópteros hacen «joint venture» con operadores turísticos y hoteles, para poder proporcionar un impulso al sector turismo.

El Estado se organiza para permitir que los centros de recarga de combustible y terminales de pasajeros y carga se encuentren diseminados en diferentes partes periféricas de la ciudad.

\section{«Buscando Estoy}

En éste escenario, el Estado peruano, comprende la necesidad faltante y da las condiciones de manera parcial para la facilitación de la operación de las aeronaves de transporte aéreo vertical.

El Sector Turismo se ve disminuido por la falta de incentivos y competividad del mercado nacional.

No se da el liderazgo y promoción por parte del gobierno al sector turismo y las empresas aéreas de transporte vertical urbano tienen cierta dificultad de conseguir clientes. El sistema se fortalece muy lentamente.

Las autoridades ponen trabas burocráticas y exigencias innecesarias a las empresas, dando como resultado el cierre de algunas de ellas.

\section{«Helicópteros Raca»}

En éste escenario, el Estado peruano, comprende a medias las necesidades faltantes y sólo da algunas de las condiciones de manera incompleta para la facilitación de la operación de las aeronaves de transporte aéreo vertical; creando barreras que dificulta la operación aérea urbana.

El Estado da apoyo a la afluencia del turismo, pero no llega a entender que el sector aeronáutico y energético debe de modernizarse, lo cual origina la obsolescencia de gran parte de la flota, originando retrasos en los vuelos solicitados e indignación de los usuarios.
No se facilita la creación de terminales de pasajeros ni de centro de recarga de combustible, limitándose las operaciones.

\section{«Me quedo en Tierra»}

En éste escenario, el Estado peruano, permanece indiferente y no comprende absolutamente de la importancia de cubrir la necesidad faltante y se opone a crear las condiciones de manera parcial para la facilitación de la operación de las aeronaves de transporte aéreo vertical. Ésta situación es la que actualmente se experimenta en el país.

No se cuenta con normas ni reglamentación aeronáutica adecuadas y no se cumple con las normas de diseño urbanístico que permiten las condiciones más seguras para la operación aérea (no cables aéreos y áreas libres en los parques y jardines), no se cuenta con las facilidades de terminales de pasajeros y carga como de puntos de recarga de combustible, etc.

Los inversionistas de empresas de aeronaves de transporte aéreo vertical, son renuentes e inseguros de invertir en la operación en el cielo de Lima.

\section{Matriz AHP [19]}

Se empleó la herramienta del análisis de la Matriz AHP (Analytic Hierarchy Process), que es un proceso de Análisis Jerárquico diseñado para resolver problemas complejos de criterios múltiples y se usa para la toma de decisiones (Wilches-Chaux, 2001).

Éste proceso requiere proporcionar evaluaciones subjetivas respecto a la importancia relativa de cada uno de los criterios especificando la preferencia con respecto a las alternativas y criterios. Como resultado del AHP se jerarquiza con prioridades de preferencia para las alternativas de decisión. 
La herramienta AHP de los cuadros anteriores, nos confirma que el escenario planteado por el grupo de expertos «Two Thumps Up» es el escenario apuesta. Resalta como un factor muy importante la afluencia turística, seguido por la eficiencia del aparato estatal.

Figura 13. Resultado de la evaluación de escenarios AHP

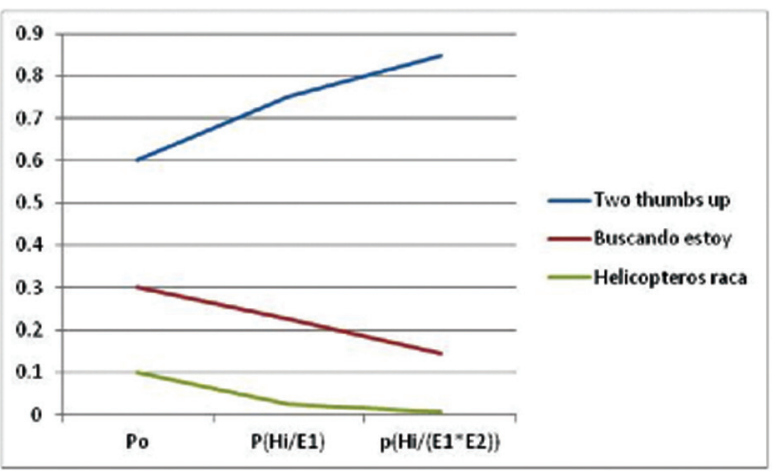

Con éste análisis realizado con la teoría bayesiana, se comprueba la validez del escenario meta planteado con respecto a los otros escenarios, relacionados a los diferentes variables.

\section{Conclusiones}

\section{Peligro y Riesgo Latente en Lima.}

La ciudad de Lima con un promedio de 10 Millones de habitantes, está con la amenaza latente de múltiples fenómenos sismológicos, climatológicos, etc., así como producto del hombre.

\section{Espacio Aéreo de Lima no es utilizado}

La ciudad de Lima cuenta con su espacio aéreo el cual aún no es aprovechado para el transporte de personas y carga.

\section{Falta de Cultura de Transporte Aéreo Vertical}

Las instituciones públicas del gobierno y las privadas en la ciudad de Lima, aún no consideran el empleo de aeronaves para el transporte aéreo vertical.

\section{Escenario Apuesta: «Two Thumbs Up»}

El Resultado de los análisis del proceso de jerarquía de escenarios AHP arroja como resultado que el escenario apuesta es el que más convendría. Teniendo en cuenta los factores Turísticos y de Eficiencia del Estado.

\section{Recomendaciones}

\section{Implementación Integral del Escenario} Apuesta con los Planes Multisectoriales (INDECI, MTC, MINDEF, PCM, etc.).

Se recomienda que los Planes Estratégicos a realizarse sean articulados con los Planes de los diferentes sectores tales como del INDECI, MINDEF, PCM, Regiones, Municipios, etc. Con la finalidad de que sean aprovechados por toda la ciudadanía,

\section{Control del Espacio Aéreo en LIMA}

Se recomienda que el Congreso de la República del Perú legisle la creación de un ente controlador del espacio aéreo tal cómo se hace en la Ciudad de Sao Paulo, Brasil, la cual está a cargo de la Fuerza Aérea.

Asimismo, se recomienda que el Congreso legisle la creación de una institución autónoma privada que se haga responsable del control de las habilitaciones de las diferentes compañías de que se adhieran a la oferta del servicio del Transporte Aéreo Vertical Urbano.

\section{Promoción de la Cultura Aeronáutica}

Se recomienda que la Promoción de la Cultura Aeronáutica en la población sea liderado por el Presidente de la República del Perú, a fin sea explicado sobre la conveniencia de contar con éste sistema y sea recibido de buena manera por las autoridades y la población reacia al cambio y a la modernidad. 


\section{Conectividad del Turismo y Eficiencia del Estado}

La implementación del Modelo del Sistema de Transporte Aéreo Vertical debe de estar dirigido a solucionar las deficiencias de conectividad en el sector Turismo y de Eficiencia del Estado y respaldado por normatividad que facilite una operación segura y rentable con aeronaves de última tecnología, tanto para las empresas como para el público usuario.

\section{Referencias}

Campos Lizarzaburu, W. 2010. Matriz de Consistencia.

CNN, (2010). Los ricos de Sao Paulo esquivan el tráfico con Helicópteros.

Congreso de la República del Perú, (2000). Ley de Aeronáutica Civil del Perú. Diario Oficial El Peruano. Extraído el 27 Febrero 2012 desde: http://www.mtc.gob.pe/portal/ transportes/aereo/normaslegales/docs/PDF/Ley de Aeronautica Civil 27261.pdf

Fernández Güell, JM. (2001). Creación de Escenarios, El diseño de los escenarios en el ámbito empresarial. Madrid: Ediciones Pirámide.

Gutiérrez, J. Coronel FAP (r) (1982). Manual del Helicóptero de Combate de la FAP.

Gutiérrez, J. y Carbonel, G. 2011.InkaKopter, Transporte Aéreo de Turismo VIP.

Gutiérrez, J. (2010). Creación de la Escuela de Helicópteros Helischool.

Harrison, K. (2013). América Latina un Mercado en Ascenso para los helicópteros. AIN On Line. http:// www.ainonline.com/aviation-news/labace-conventionnews/2013-08-12/america-latina-e-um-mercado-em-ascensao-para-helicopteros
López, M. y Mojíca, F. (2004). Estudio Prospectivo y Estratégico Municipio de Fusagasuga.

Michel, G. y Phillipe, D. (2009). Encuesta Delphi, Lipsor, «La Prospectiva Estratégica». Cap II.

Ministerio de Transportes y Comunicaciones (2010). MTC Relación de Empresas Aéreas del Perú. MTC. Extraído el 18 de Febrero 2012 de fuente propia de Internet desde: http://www.mtc.gob.pe/portal/transportes/aereo/ aeronauticacivil/emp nac/EmpNacionales.pdf

Ministerio de Transporte y Comunicaciones. (2001, 09 de Agosto). DS N" 016-2007-MTC Reglamento de la Ley de Aeronáutica Civil del Perú. Extraído el 07 de Febrero 2012 desde fuente: propia de Internet.

Ortega, F. (2010). Polígono STEEP-VOC, MBA.

Presidente de la República, (2006, 29 Enero). Reglamento de Ley de Seguridad de la Aviación Civil D.S. 007-2006-MTC. Diario Oficial El Peruano Normas Legales pág.: 311316 . Extraído el 08 de Febrero 2012 desde fuente: propia de Internet.

Roubelat, F. Structural analysis with the MICMAC Method \& Actors' Strategy with MACTOR Method, Millenium Project Arcade (J), Sirius, Godet (M), CNAM, Meunier (F), CNAM.

Terrádez, MG. Análisis de Componentes Principales, Matriz Porcentual de Variables.

Saaty, Th. (2001). Matriz de AHP "Analytical Hierarchy Process", (1980),.Control Toolbox (6.0), User's Guide, The Math Works, pp. 2-10-2-35.

Wilches-Chaux, G. 2001. Vulnerabilidad, extraído de Parés.

Wilches-Chaux, G. (2001). Desastre, extraído de Parés. 